\title{
Beiträge zur Chemie des Phosphors, 68 $^{1}$ Tri-t-butyl-cyclotriphosphan
}

\author{
Contributions to the Chemistry of Phosphorus, $68^{1}$ \\ Tri-t-butyl-cyclotriphosphane
}

\section{Marianne Baudler, Josef Hahn, Hermann Dietsch und Gabriele Fürstenberg}

Institut für Anorganische Chemie der Universität Köln

Professor Leonhard Birkofer zum 65. Geburtstage gewidmet

(Z. Naturforsch. 31 b, 1305-1310 [1976]; eingegangen am 28. Juni 1976)

Tri-t-butyl-cyclotriphosphane, Cyclotriphosphanes, Triphosphiranes, 1,2-Di-t-butyl-1,2-bis(trimethylsilyl)-diphosphane, Cyclotetraphosphanes

\begin{abstract}
In the reaction of magnesium with trimethylchlorosilane and $t$-butyldichlorophosphane the hitherto unknown tri-t-butyl-cyclotriphosphane, $(t-\mathrm{BuP})_{3}(\mathbf{1})$, is produced besides the partially silylated phosphanes $t$ - $\mathrm{BuP}\left(\mathrm{SiMe}_{3}\right)_{2}$ and $(t-\mathrm{BuPSiMe})_{2}$ as well as the cyclotetraphosphane $(t-\mathrm{BuP})_{4}$. Shortly after the beginning of the reaction the amount of 1 is about 50 mole- $\%$ and decreases during the reaction time. Thermally and in solution the $\mathrm{P}_{3}$ ring compound (1) is surprisingly stable and can be isolated in a pure state by fractional distillation. $(t \text {-BuP })_{3}$ clearly differs from the oligomeric $(t-\mathrm{BuP})_{4}$ by the lower melting point, the molar mass and by the ${ }^{31} \mathrm{P}$ NMR and IR spectra. The $t$-butyl groups in 1 are situated above and below the $\mathrm{P}_{3}$ ring plane.
\end{abstract}

In Fortsetzung unserer Untersuchungen über organylsubstituierte Cyclotriphosphane (Triphosphirane $)^{1-3}$ konnten wir die Bildung von Tri-t-butylcyclotriphosphan, $(t-\mathrm{BuP})_{3}(\mathbf{1})$, bei der Darstellung von $(t \text {-BuPSiMe })_{2}$ und $t$ - $\mathrm{BuP}\left(\mathrm{SiMe}_{3}\right)_{2}$ beobachten und dieses Cyclotriphosphan als thermisch überraschend beständige Verbindung isolieren.

\section{Darstellung von $(t-\mathrm{BuP})_{3}(\mathbf{1})$}

Geeignete Ausgangsverbindungen für die Synthese von Triorganyl-cyclotriphosphanen aus Diphosphan- und Monophosphanderivaten nach der allgemeinen Reaktionsgleichung

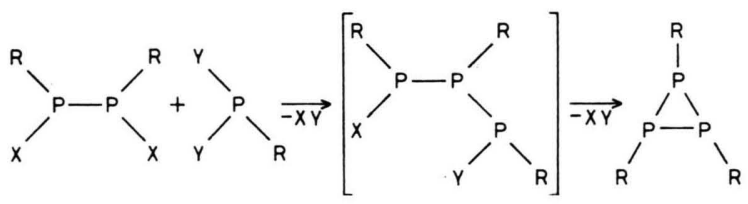

Sonderdruckanforderungen an Prof. Dr. M. BAUDLER, Institut für Anorg. Chemie der Universität Köln, Greinstraße 6, D-5000 Köln 41. sind bis-silylierte Phosphane der Zusammensetzungen $\mathrm{Me}_{3} \mathrm{Si}(\mathrm{R}) \mathrm{P}-\mathrm{P}(\mathrm{R}) \mathrm{SiMe}_{3}$ und $\mathrm{RP}\left(\mathrm{SiMe}_{3}\right)_{2}$. So konnten Triphenyl-cyclotriphosphan, $(\mathrm{PPh})_{3}$, und Diphenyl-äthyl-cyclotriphosphan, (PPh $)_{2} \mathrm{PEt}$, ausgehend vom 1.2-Diphenyl-1.2-bis(trimethylsilyl)diphosphan ${ }^{4}$ durch Reaktion mit Phenyldichlorphosphan bzw. Äthyldichlorphosphan gewonnen werden ${ }^{1}$. Im Hinblick auf die bekannte Stabilitätserhöhung kleiner Ringsysteme durch großvolumige Substituenten $R$ waren entsprechende Cyclisierungen mit 1.2-Di-t-butyl-1.2-bis(trimethylsilyl)-diphosphan, $\mathrm{Me}_{3} \mathrm{Si}(t-\mathrm{Bu}) \mathrm{P}-\mathrm{P}(t-\mathrm{Bu}) \mathrm{SiMe}_{3}$ (2), und $t$-Butylbis(trimethylsilyl)-phosphan, $t$ - $\mathrm{BuP}\left(\mathrm{SiMe}_{3}\right)_{2}(3)$, von Interesse. Diese Verbindungen waren kürzlich von Schumann und Mitarb. ${ }^{5,6}$ durch eine Eintopfreaktion aus Magnesium, Trimethylchlorsilan und $t$-Butyldichlorphosphanin den Molverhältnissen 1,5: $1: 1$ bzw. 2: 2: 1 (mehrtägiges Erhitzen in Tetrahydrofuran) dargestellt worden. Dabei wurde im ersten Fall neben dem Diphosphan (2) noch die Bildung von Tetra-t-butyl-cyclotetraphosphan, $(t \text {-BuP })_{4}(4)$, beobachtet ${ }^{5}$. 
Beim Nacharbeiten der Darstellungsvorschriften für die Verbindungen $\mathbf{2}$ und $\mathbf{3}$ stellten wir fest, daß jeweils eine Reihe weiterer Substanzen gebildet wird, wodurch die Reinisolierung von 2 bzw. 3 erheblich erschwert wird. Da eine dieser Verbindungen im ${ }^{31} \mathrm{P}-\mathrm{NMR}$-Spektrum ein $\mathrm{A}_{2} \mathrm{~B}$-System im Bereich kettenförmiger ${ }^{7}$ oder auch cyclischer ${ }^{1}$ Triphosphane zeigt, haben wir die Reaktion von Magnesium, Trimethylchlorsilan und $t$-Butyldichlorphosphan in den angegebenen stöchiometrischen Verhältnissen ${ }^{31} \mathrm{P}-\mathrm{NMR}$-spektroskopisch näher untersucht.

Wir fanden, daß in dem betreffenden Reaktionsgemisch im wesentlichen vier Verbindungen vorliegen:

Tri-t-butyl-cyclotriphosphan (1) (siehe weiter unten; $\mathrm{A}_{2} \mathrm{~B}$-System im Bereich von -67 bis $-115 \mathrm{ppm}^{8}$ );

1.2-Di-t-butyl-1.2-bis(trimethylsilyl)-diphosphan (2) (Singulett bei $\delta=-63,9 \mathrm{ppm}^{5}$ );

$t$-Butyl-bis(trimethylsilyl)-phosphan (3) (Singulett bei $\delta=-108,9 \mathrm{ppm})$;

Tetra-t-butyl-cyclotetraphosphan (4) (Singulett bei $\delta=-58,1 \mathrm{ppm}^{9}$ ).

Daneben sind unmittelbar nach Reaktionsbeginn noch einige AB-Systeme beobachtbar, die jedoch nach wenigen Stunden nicht mehr erscheinen. Aufgrund der chemischen Verschiebungen dürfte es sich um chlorhaltige, unsymmetrisch $t$-butyl- oder/und trimethylsilyl-substituierte Diphosphane handeln. $t$-Butyldichlorphosphan (Singulett bei $\delta=+198$ ppm) läßt sich bereits kurz nach beendeter Zugabe nicht mehr nachweisen.

Das Verhältnis der Konzentrationen der einzelnen Reaktionsprodukte untereinander ist von verschiedenen Faktoren abhängig. Erwartungsgemäß wird bei den Silylphosphanen das Produktverhältnis $2: 3$ im wesentlichen durch die angewandte Stöchiometrie bestimmt. Die Relation der Gesamtmenge an $\mathbf{2}$ und 3 zu der Gesamtmenge an den Cyclophosphanen 1 und 4 ist dagegen entscheidend von der Geschwindigkeit der Zugabe des $t$-Butyldichlorphosphans und der Nachreaktionszeit abhängig. Schnelles Eintropfen begünstigt die Bildung der cyclischen Verbindungen; durch langsame Zugabe kann die Ausbeute an 2 bzw. 3 (je nach eingesetzter Stöchiometrie) erhöht werden.

Die Konzentrationen der einzelnen Reaktionsprodukte in Abhängigkeit von der Reaktionszeit sind für einen ausgewählten Ansatz der Stöchiometrie $\mathrm{Mg}: \mathrm{Me}_{3} \mathrm{SiCl}: t-\mathrm{BuPCl}_{2}=1,5: 1: 1$ in Tab. I angegeben. Ganz entsprechende Ergebnisse werden
Tab. I. Konzentrationen (in Mol-\%) der Reaktionsprodukte in Abhängigkeit von der Reaktionszeit $\left(\mathrm{Mg}: \mathrm{Me}_{3} \mathrm{SiCl}: t-\mathrm{BuPCl}_{2}=1,5: 1: 1 ;\right.$ Zutropfzeit von $t$ - $\mathrm{BuPCl}_{2} \approx 1,5 \mathrm{Stdn}$.).

\begin{tabular}{llllll}
\hline $\begin{array}{l}\text { Reaktionszeit } \\
\text { nach Zugabe } \\
\text { von } t \text {-BuPCl } \text {-Bh] }[\mathrm{L}\end{array}$ & $\mathbf{2}$ & $\mathbf{3}$ & $\mathbf{4}$ & $\begin{array}{l}\text { Unsymmetr. } \\
\text { Diphosphane } \\
\text { (AB-Systeme) }\end{array}$ \\
\hline 0,5 & 48,1 & 5,9 & - & 11,0 & 35,0 \\
14 & 47,0 & 29,4 & 11,7 & 11,7 & - \\
19 & 44,9 & 34,4 & 6,9 & 13,8 & - \\
36 & 42,0 & 38,3 & 3,8 & 15,7 & - \\
45 & 42,0 & 38,3 & 3,8 & 15,7 & - \\
70 & 42,8 & 35,7 & 3,6 & 17,8 & - \\
91 & 36,7 & 33,3 & 13,4 & 16,6 & - \\
\hline
\end{tabular}

auch bei dem Reaktantenverhältnis $2: 2: 1$ erhalten.

Insgesamt ergibt sich für die Reaktion von Magnesium mit Trimethylchlorsilan und $t$-Butyldichlorphosphan folgendes:

1. Die qualitative Zusammensetzung des Produktgemisches ist weitgehend unabhängig von der eingesetzten Stöchiometrie. Die quantitative Produktverteilung hängt - außer von der Stöchiometrie - ganz wesentlich von der Zugabegeschwindigkeit des $t$-Butyldichlorphosphans und der Reaktionszeit ab.

2. Der Anteil an Tri-t-butyl-cyclotriphosphan (1) ist unmittelbar nach Zugabe des $t$-Butyldichlorphosphans am größten und nimmt mit zunehmender Umsetzungsdauer langsam ab.

3. Die Konzentration an Tetra-t-butyl-cyclotetraphoshan (4) ändert sich im Verlauf der Reaktion nur geringfügig.

4. Tri-t-butyl-cyclotriphosphan - und möglicherweise auch Tetra-t-butyl-cyclotetraphosphan reagieren mit Magnesium und Trimethylchlorsilan weiter zu 1.2-Di-t-butyl-1.2-bis(trimethylsilyl)-diphosphan (2) und $t$-Butyl-bis(trimethylsilyl)-phosphan (3), die zu Beginn der Reaktion auch aus den chlorhaltigen unsymmetrischen Diphosphanen gebildet werden. Für 2 und $\mathbf{3}$ wird ein Ausbeutemaximum bei entsprechender Stöchiometrie schon nach ungefähr 36 bzw. 20 Stunden erreicht (Reaktionszeit für 3 nach ScHUMANN und Rösch ${ }^{6} 6$ Tage).

Die quantitative Auftrennung und Isolierung der einzelnen Reaktionsprodukte bereitete zunächst Schwierigkeiten.

Versuche zur fraktionierten Destillation über eine Vigreuxkolonne (15 bzw. $30 \mathrm{~cm}$ ) bei gleichzeitiger 
${ }^{31} \mathrm{P}-\mathrm{NMR}$-spektroskopischer Kontrolle der erhaltenen Fraktionen ergaben, daß nur das leichtflüchtige Monophosphan $\mathbf{3}$ in der ersten Fraktion (Siedetemperatur etwa $45-58{ }^{\circ} \mathrm{C} / 0,1$ Torr) rein anfällt. Die zweite Fraktion (etwa $75-95{ }^{\circ} \mathrm{C}$ ) enthält die Hauptmenge des Cyclotriphosphans (1) sowie außerdem noch merkliche Anteile von Diphosphan (2). In der dritten Fraktion (etwa $95-110^{\circ} \mathrm{C}$ ) findet sich hauptsächlich $\mathbf{2}$; daneben liegen 4 , Reste von 1 und geringe Mengen einer unbekannten Substanz (ABSystem mit $\delta_{\mathrm{A}}=\mathbf{- 4 , 5} \mathrm{ppm}, \delta_{\mathrm{B}}=-128,0 \mathrm{ppm}$, $J_{\mathrm{AB}}=325,5 \mathrm{~Hz}$ ) vor. Letztere Verbindung ist auch im Destillationsrückstand enthalten, der im wesentlichen aus 4 - neben restlichen Anteilen von 2 besteht.

Die vollständige Auftrennung des Substanzgemisches gelang durch fraktionierte Destillation über eine $30 \mathrm{~cm}$-Spaltrohrkolonne ${ }^{10}$ bei 0,2 Torr :

1. Fraktion (Temperatur am Kolonnenkopf 52$\left.56{ }^{\circ} \mathrm{C}\right)$ :

$t$-Butyl-bis(trimethylsilyl)-phosphan (3); farblose, an der Luft selbstentzündliche Flüssigkeit.

2. Fraktion $\left(76-80{ }^{\circ} \mathrm{C}\right)$ :

Tri-t-butyl-cyclotriphosphan (1); nach der Destillation farblose Flüssigkeit, die beim Abkühlen zu nadelförmigen Kristallen erstarrt.

3. Fraktion $\left(103-108^{\circ} \mathrm{C}\right)$ :

1.2-Di-t-butyl-1.2-bis(trimethylsilyl)-diphosphan (2); farblose, an der Luft selbstentzündliche Flüssigkeit.

4. Destillationsrückstand (gelbbrauner Feststoff, der teilweise in die Kolonne sublimiert):

Tetra-t-butyl-cyclotetraphosphan (4) neben Diphosphan (2) und Thermolyseprodukten.

Auf diesem Wege ist es möglich, reines Tri-t-butylcyclotriphosphan (1) in einer Ausbeute von 31\% (nähere Einzelheiten siehe unter ,Experimentelles“") zu gewinnen. 1.2-Di-t-butyl-1.2-bis(trimethylsilyl)diphosphan (2) kann ebenfalls vollkommen rein in 19-proz. Ausbeute (modifizierte Reaktionsbedingungen, siehe „Experimentelles“) erhalten werden.

\section{Eigensehaiten und spektroskopische Untersuchung von 1}

Die farblosen, nadelförmigen Kristalle von 1 (Schmp. $41{ }^{\circ} \mathrm{C}$, geschl. Rohr) sind bei $-18{ }^{\circ} \mathrm{C}$ unter Inertgas monatelang beständig. Sie lösen sich in Benzol, Toluol, Tetrahydrofuran,'Dioxan, Schwefelkohlenstoff und $n$-Pentan, während mit halogenier- ten Kohlenwasserstoffen oder Wasser Zersetzung eintritt. Durch Luftsauerstoff wird 1 verhältnismäßig rasch oxydativ angegriffen, so da $\beta$ in feiner Verteilung oder bei Kontakt mit Zellstoff Selbstentzündung eintritt. Die Verbindung wurde durch Elementaranalyse, Molmasse-Bestimmung, ${ }^{31} \mathrm{P}-\mathrm{NMR}$-, ${ }^{1} \mathrm{H}-\mathrm{NMR}$-, IR- und Massenspektrum charakterisiert.

Die Molmasse ergab sich (osmometrisch in $\mathrm{CS}_{2}$ bei $25^{\circ} \mathrm{C}$ ) im Mittel zu 259 (theor. für $\left.(t-\mathrm{BuP})_{3}: 264,3\right)$. In Übereinstimmung damit entspricht im Massenspektrum (20 eV, ohne Zusatzheizung) die Signalgruppe mit den höchsten Massenzahlen (Hauptpeak $m / e$ 264, $\left.\mathrm{I}_{\text {rel }} 33\right)$ dem Molekülion $(t-\mathrm{BuP})_{3}{ }^{+}$. Neben dem Basispeak für $\mathrm{C}_{4} \mathrm{H}_{9}+(57,100)$ sind in dem auffallend linienarmen Spektrum außerdem noch die Fragmentionen $\mathrm{M}^{+}{ }_{-} \mathrm{C}_{4} \mathrm{H}_{9}(207,15), \mathrm{M}^{+}{ }_{-} 2 \mathrm{C}_{4} \mathrm{H}_{9}$ (150, 13) und die daraus durch Wasserstoffeinfang gebildeten Ionen $(t-\mathrm{Bu})_{2} \mathrm{P}_{3} \mathrm{H}^{+}(208,72), t-\mathrm{BuP}_{3} \mathrm{H}^{+}(151$, $82), t-\mathrm{BuP}_{3} \mathrm{H}_{2}+(152,87)$ mit merklicher Intensität beobachtbar. Hinweise auf die Signalgruppe für das Ion $(t \text {-BuP })_{4}{ }^{+}(352)$ konnten auch bei Erhöhung der Registrierempfindlichkeit um den Faktor 3 nicht gefunden werden. Demnach weist das $\mathrm{P}_{3}$-Ringgerüst von 1 gegenüber Fragmentierung und Ringumlagerung eine bemerkenswerte Stabilität auf.

Das ${ }^{31} \mathrm{P}\left\{{ }^{1} \mathrm{H}\right\}$-NMR-Spektrum von 1 (siehe Abb. 1) zeigt 8 Signale in den charakteristischen Abstandsrelationen eines $\mathrm{A}_{2} \mathrm{~B}$-Systems. Ein Hinweis auf das gleichzeitige Vorliegen von 4 (Singulett bei $\delta=-58$ $\mathrm{ppm}^{9}$ ) ist in den Spektren der Reinsubstanz nicht $\mathrm{zu}$ finden. Aus dem Auftreten des $\mathrm{A}_{2} \mathrm{~B}$-Systems ergibt sich, da $\beta(t-B u P)_{3}$ in dem Strukturisomer vor-

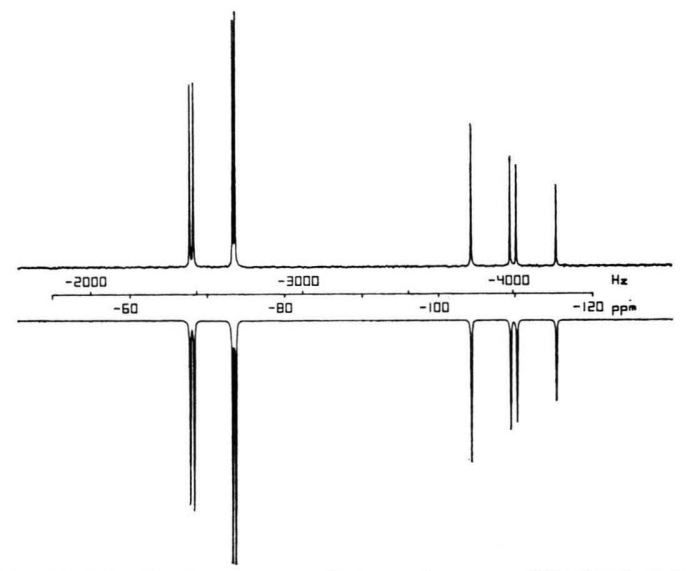

Abb. 1. Beobachtetes und berechnetes ${ }^{31} \mathrm{P}\left\{{ }^{1} \mathrm{H}\right\}-\mathrm{PFT}$ Kernresonanzspektrum von $(t \text {-BuP })_{3} \quad(\mathbf{1})$ in $\mathrm{C}_{6} \mathrm{D}^{9}$ ( $\approx 50$-proz.) bei $33{ }^{\circ} \mathrm{C}$. 
liegt, bei dem zwei tert-Butylgruppen ober- bzw. unterhalb der Phosphordreiring-Ebene und eine auf der jeweils entgegengesetzten Seite angeordnet sind. Das andere Isomer, in dem alle drei Substituenten auf der gleichen Ringseite liegen, ist offensichtlich thermodynamisch instabil.

Bei der Analyse des Spektrums wurden die in Tab.II angegebenen NMR-Parameter erhalten. Das mit diesen Daten berechnete Spektrum ist dem beobachteten in Abb. 1 gegenübergestellt.

Tab. II. Chemische Verschiebungen und Kopplungskonstante von $(t \text {-BuP })_{3}$ in $\mathrm{C}_{6} \mathrm{D}_{6} \quad(\approx 50$-proz. $)$ bei $33{ }^{\circ} \mathrm{C}^{*}$.

\begin{tabular}{lll}
\hline$\delta_{\mathrm{A}_{2}}$ & $\delta_{\mathrm{B}}$ & $J_{\mathrm{AB}}$ \\
\hline$-71,0$ & $-109,6$ & 201,1 \\
$(-2587,1)$ & $(-3992,0)$ & \\
\hline
\end{tabular}

* $\delta$-Werte in ppm gegen 85-proz. $\mathrm{H}_{3} \mathrm{PO}_{4}$ (in Klammern Werte in $\mathrm{Hz}$ relativ zur $\mathrm{H}_{3} \mathrm{PO}_{4}$-Resonanzfrequenz $36,434064 \mathrm{MHz})$; Kopplungskonstante in $\mathrm{Hz}$.

Die ermittelten chemischen Verschiebungen der $\mathrm{P}_{\mathrm{A}^{-}}$und $\mathrm{P}_{\mathrm{B}}$-Atome grenzen sich deutlich von dem für $(t-\mathrm{BuP})_{4}$ beobachteten Wert $\left(\delta=-58 \mathrm{ppm}^{9}\right)$ ab. Dies steht im Einklang mit der Erfahrung, daß bei den Cyclophosphanen $(\mathrm{PR})_{n}$ eine signifikante Abhängigkeit der chemischen Verschiebung von der Ringgröße $\mathrm{n}$ besteht ${ }^{11,12}$. Die für 1 beobachtete Hochfeldverschiebung im Vergleich zu 4 ist zwanglos als ,Effekt des kleinen Ringes“ im Cyclotriphosphanderivat $^{1} \mathrm{zu}$ interpretieren. Bemerkenswert ist jedoch, daß der Unterschied zwischen der $\mathrm{P}_{4^{-}}$und der $\mathrm{P}_{3}$-Ringverbindung bei den tert-Butylderivaten wesentlich kleiner $(\approx 30 \mathrm{ppm})$ als bei den entsprechenden Phenyl-cyclophosphanen ${ }^{1,11}(\approx 90 \mathrm{ppm})$ ist. Während das ${ }^{31} \mathrm{P}-\mathrm{NMR}$-Signal von 4 im normalen Bereich der Cyclotetraphosphane ( -48 bis $-75 \mathrm{ppm}^{11}$ ) liegt, erscheint demnach das $\mathrm{A}_{2} \mathrm{~B}$ System von $\mathbf{1}$ - auch im Vergleich mit den anderen bisher bekannten Phosphor-Dreiringverbindungen $\mathrm{P}_{3} \mathrm{Ph}_{2} \mathrm{Et}^{1}$, $\left(\mathrm{c}-\mathrm{C}_{6} \mathrm{H}_{11} \mathrm{P}\right)_{3}{ }^{13}$ und $(\mathrm{PEt})_{3}{ }^{11}$ - bei ungewöhnlich tiefem Feld. In diesem Zusammenhang ist aber zu berücksichtigen, daß beim $t-\mathrm{Bu}_{4} \mathrm{P}_{2}{ }^{9}$ und $t$ - $\mathrm{Bu}_{3} \mathrm{P}^{14}$ ebenfalls auffällige Tieffeldverschiebungen im Vergleich mit anderen Tetraorganyl-diphosphanen bzw. tertiären Phosphanen beobachtet worden sind. Offensichtlich bewirken die tert-Butylgruppen immer dann eine deutliche Entschirmung der PKerne, wenn sterische Behinderungen der Substituenten untereinander vorliegen, was bei 4 mit „all-trans"-Konfiguration nicht der Fall ist.
Das ${ }^{1} \mathrm{H}\left\{{ }^{31} \mathrm{P}\right\}$-NMR-Spektrum von 1 (5-proz. in $\mathrm{C}_{6} \mathrm{H}_{6}, 33^{\circ} \mathrm{C}$ ) zeigt erwartungsgemäß zwei Singuletts im Intensitätsverhältnis $2: 1$ bei $\delta=1,27$ und 1,03 ppm, die den unterschiedlich angeordneten tertButylgruppen entsprechen. Bei Nicht-Entkopplung der Phosphorkerne beobachtet man ein Spektrum höherer Ordnung, in dem für die eine trans-ständige tert-Butylgruppe angenähert ein Dublett von Tripletts erscheint.

Das IR-Spektrum von 1 weist im Wellenzahlenbereich unterhalb $1200 \mathrm{~cm}^{-1}$ einige signifikante Unterschiede gegenüber dem von 4 auf (siehe Abb.2). So zeigt die Bandengruppe für die inneren Schwingungen der $t$-Butylgruppen zwischen 1100 und 1200 $\mathrm{cm}^{-1}$ einen deutlich anderen Habitus (Fehlen der Absorption bei $1125 \mathrm{~cm}^{-1}$, zusätzliche Schulter bei $1192 \mathrm{~cm}^{-1}$ ). Außerdem ist die intensive Doppelbande von 4 bei $879 / 889 \mathrm{~cm}^{-1}$ im Spektrum von 1 nicht vorhanden. Weiterhin erscheint im P-C-Valenzschwingungsbereich um $600 \mathrm{~cm}^{-1}$ bei 1 nur eine mittelstarke Bande bei $572 \mathrm{~cm}^{-1}$ mit einer Schulter bei $583 \mathrm{~cm}^{-1}$, während bei 4 zwei nahezu gleichstarke Absorptionen bei 577 und $616 \mathrm{~cm}^{-1}$ auftreten. Am auffallendsten ist jedoch im Spektrum von 1 das Vorhandensein einer zusätzlichen mittelstarken Bande bei $322 \mathrm{~cm}^{-1}$, während die längerwellige Absorption von 4 bei $273 \mathrm{~cm}^{-1}$ fehlt. Insgesamt ist damit IR-spektroskopisch eine einfache Differenzierung der oligomeren $t$-Butyl-cyclophosphane $\mathbf{1}$ und 4 - auch in Gemischen - möglich.

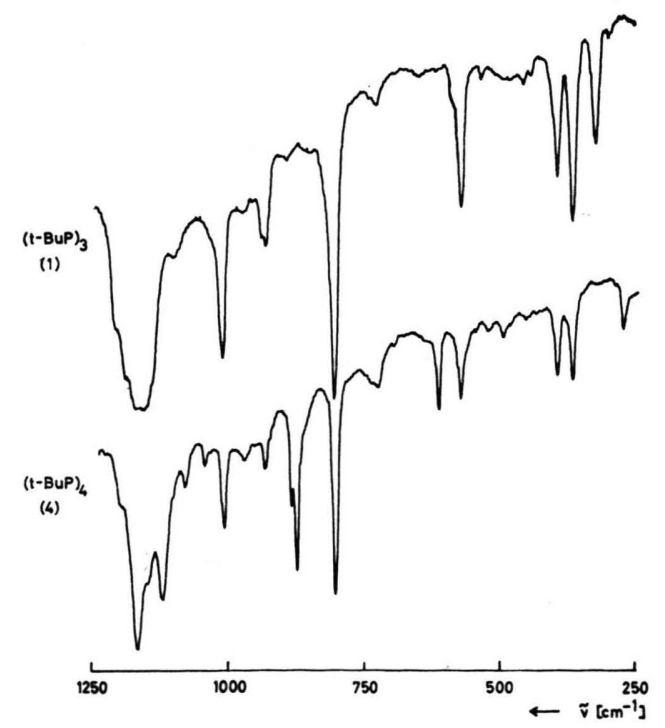

Abb. 2. IR-Spektren (Ausschnitt) von $(t-\mathrm{BuP})_{3}$ (1) und $(t-\mathrm{BuP})_{4}(4)$. 


\section{Diskussion der Ergebnisse}

Mit der Verbindung 1 ist die Darstellung eines thermisch überraschend beständigen Cyclotriphosphans (Triphosphirans) gelungen. Der beobachtete Schmelzpunkt von $41{ }^{\circ} \mathrm{C}$ ist reversibel, und auch bei der Destillationstemperatur von $76-80^{\circ} \mathrm{C} / 0,2$ Torr findet offensichtlich keine nennenswerte Umlagerung des Phosphor-Dreiringgerüstes statt. In Lösung bei Raumtemperatur ist $\mathbf{1}$ - im Unterschied zu anderen Cyclotriphosphanen ${ }^{1}$ - gleichfalls bemerkenswert beständig. So kann zum Beispiel in Tetrahydrofuran ( $\approx 5$-proz., strengster Luft- und Feuchtigkeitsausschluß) trotz der Polarität dieses Solvens auch nach 14 Tagen ${ }^{31} \mathrm{P}-\mathrm{NMR}$-spektroskopisch noch kein Anzeichen für die Bildung von 4 gefunden werden. Die erstaunlich große thermische und kinetische Stabilität von 1 ist zweifellos auf die besondere sterische Situation der $t$-Butyl-Substituenten zurückzuführen, die zum einen die Energie des $\mathrm{P}_{3}$-Ringsystems erniedrigen und zum anderen die Reaktivität der freien Elektronenpaare an den Phosphoratomen erheblich einschränken. Eine ähnlich große stabilitätserhöhende Wirkung auf das Phosphor-Dreiringgerüst haben wir kürzlich auch für den CyclohexylSubstituenten festgestellt ${ }^{13}$.

Die Bildung von 1 bei der Reaktion von Magnesium mit Trimethylchlorsilan und $t$-Butyldichlorphosphan erfolgt sehr wahrscheinlich überwiegend durch direkte Enthalogenierung von $t$-Butyldichlorphosphan ohne Beteiligung von Trimethylchlorsilan:

$$
\begin{aligned}
t-\mathrm{BuPCl}_{2}+\mathrm{Mg} \underset{-\mathrm{MgCl}_{2}}{\longrightarrow} & \frac{1}{n}(t-\mathrm{BuP})_{n} \\
1 & : n=3 \\
& 2: n=4
\end{aligned}
$$

In Übereinstimmung damit konnten wir das Auftreten von 1 neben 4 inzwischen auch bei der Umsetzung von $t$-Butyldichlorphosphan mit Magnesium bzw. anderen Metallen in Abwesenheit von Trimethylchlorsilan feststellen ${ }^{15}$. Die alternativen Reaktionsfolgen über $t$-Butyltrimethylsilyl-chlorphosphan bzw. das Diphosphan (2) als Zwischenstufen

$$
\begin{gathered}
t-\mathrm{BuPCl}_{2}+\mathrm{Me}_{3} \mathrm{SiMgCl} \underset{-\mathrm{MgCl}_{2}}{\longrightarrow} t \text { - } \mathrm{BuP}\left(\mathrm{SiMe}_{3}\right) \mathrm{Cl} \\
\frac{1}{n}(t-\mathrm{BuP})_{n} \\
\stackrel{2-\mathrm{Me}_{3} \mathrm{SiCl}}{ } \\
\stackrel{\mathrm{Me}_{3} \mathrm{Si}(t-\mathrm{Bu}) \mathrm{P}-\mathrm{P}(t-\mathrm{Bu}) \mathrm{SiMe}_{3}+t-\mathrm{BuPCl}_{2}}{\underset{-2 \mathrm{Me}_{3} \mathrm{SiCl}}{\longrightarrow}}(t-\mathrm{BuP})_{3}
\end{gathered}
$$

dürften im Hinblick auf die kurze Reaktionszeit und die relativ niedrige Umsetzungstemperatur ${ }^{16}$ allenfalls von untergeordneter Bedeutung sein.

Die nähere Untersuchung des reaktiven Verhaltens von 1 und eine Röntgenstrukturanalyse sind im Gange.

\section{Experimentelles}

Alle Arbeiten wurden unter Ausschluß von Luft und Feuchtigkeit in gereinigter Argonatmosphäre durchgeführt. Die verwendeten Lösungsmittel waren getrocknet und Ar-gesättigt. Die Massenspektren wurden mit dem Gerät CH 5 der Fa. Varian MAT und die IR-Spektren mit dem Perkin-Elmer-GitterSpektrometer 457 aufgenommen.

Die Messung der Kernresonanzspektren erfolgte mit einem Multikern-Kernresonanzspektrometer HX $90 \mathrm{E}$ der Fa. Bruker-Physik AG, Karlsruhe. Die ${ }^{31} \mathrm{P}-\mathrm{NMR}-\mathrm{Spektren}$ wurden nach der PFTMethode aufgenommen, wobei das Magnetfeld auf das Deuterium-Signal des $\mathrm{C}_{6} \mathrm{D}_{6}$ bei $13,812 \mathrm{MHz}$ stabilisiert war, entsprechend einer ${ }^{31} \mathrm{P}-$ Resonanzfrequenz für 85-proz. $\mathrm{H}_{3} \mathrm{PO}_{4}$ von $36,434064 \mathrm{MHz}$. Die chemischen Verschiebungen sind auf 85-proz. $\mathrm{H}_{3} \mathrm{PO}_{4}$ bezogen (negatives Vorzeichen bei Hochfeldverschiebung).

Tri-t-butyl-cyclotriphosphan (1): Zu 10,6 g (0,44 Grammatom) Magnesium in $100 \mathrm{ml}$ Tetrahydrofuran tropft man in der Siedehitze unter starkem Rühren innerhalb von $30 \mathrm{Min}$. $31,6 \mathrm{~g}(0,29 \mathrm{Mol})$ Trimethylchlorsilan, vermischt mit $0,5 \mathrm{ml}$ Brom. Dabei sinkt die Siedetemperatur von $66^{\circ} \mathrm{C}$ auf $\approx 60{ }^{\circ} \mathrm{C}$ und steigt nach beendeter Zugabe langsam wieder auf $65^{\circ} \mathrm{C}$ an. Sobald die Temperatur konstant ist, wird unter weiterem Rühren und Rückflußkochen eine Lösung von 46,2 g $(0,29 \mathrm{Mol})$ $t$-Butyldichlorphosphan in $25 \mathrm{ml}$ Tetrahydrofuran innerhalb von 1-1,5 Stdn. gleichmäßig zugegeben. Anschließend läßt man 12 Stdn. in der Siedehitze nachreagieren. Nach dem Abkühlen wird der gebildete Niederschlag abgesaugt, mit Pentan gewaschen und das mit der Waschlösung vereinigte Filtrat im Wasserstrahlvakuum vom Lösungsmittel befreit. Zur Entfernung von restlichem Magnesiumchlorid versetzt man mit Pentan, filtriert erneut und zieht das Lösungsmittel i. Vak. ab. Fraktionierte Destillation des Einengrückstandes über eine $30 \mathrm{~cm}$ Spaltrohrkolonne ${ }^{10}$ ergibt 8,0 g reines 1 (Temperatur am Kolonnenkopf $76-80^{\circ} \mathrm{C} / 0,2$ Torr); Ausbeute $31 \%$, bezogen auf $t$-Butyldichlorphosphan.

$\mathrm{C}_{12} \mathrm{H}_{27} \mathrm{P}_{3}(264,3)$

Ber. C 54,54 H 10,30 P 35,16,

Gef. C 54,30 H 10,19 P 34,86.

Mol.-Masse 258,6 (osmometr. in Schwefelkohlenstoff bei $\left.25^{\circ} \mathrm{C}\right)$.

1.2-Di-t-butyl-1.2-bis (trimethylsilyl)-diphosphan (2): Wie bei 1 werden $10,1 \mathrm{~g}$ (0,42 Grammatom) Magnesium in $100 \mathrm{ml}$ Tetrahydrofuran mit $30,1 \mathrm{~g}$ $(0,28 \mathrm{Mol})$ Trimethylchlorsilan und $0,5 \mathrm{ml}$ Brom und 
danach mit 44,0 g (0,28 Mol) $t$-Butyldichlorphosphan in $25 \mathrm{ml}$ Tetrahydrofuran umgesetzt. Die Zutropfzeit für die $t$-Butyldichlorphosphan-Lösung wird auf $5^{1 / 2}$ Stdn. und die Nachreaktionszeit auf 36 Stdn. erhöht. Die Aufarbeitung und destillative Trennung erfolgt wie bei 1 . Es werden 8,5 g reines 2 (Temperatur am Kolonnenkopf $103-108^{\circ} \mathrm{C} / 0,2$ Torr)

1 67. Mitt.: M. Baudler, B. Carlsohn, B. Kloth u. D. Kосн, Z. Anorg. Allg. Chem., im Druck.

2 M. Baudler, K. KipKer u. H.-W. Valpertz, Naturwissenschaften 53, 612 [1966].

3 M. Baudler u. M. Bock, Z. Anorg. Allg. Chem. 395, 37 [1973].

4 M. Baudler, M. Hallab, A. Zarkadas u. E. Tolls, Chem. Ber. 106, 3962 [1973].

5 H. Schumann u. R. Fischer, J. Organomet. Chem. 88, C 13 [1975].

6 H. Schumann u. L. Rösch, Chem. Ber. 107, 854 [1974].

7 M. Baudler, D. Koch u. B. Carlsohn, Veröffentlichung in Vorbereitung.

8 Im Unterschied zu den bisherigen Arbeiten dieser Reihe sind Hochfeldverschiebungen durch negatives Vorzeichen gekennzeichnet.

9 K. Issleib u. M. Hoffmann, Chem. Ber. 99, 1320 [1966]. vom Schmp. $12-13,5^{\circ} \mathrm{C}$ erhalten; Ausbeute $19 \%$. Das ${ }^{31} \mathrm{P}-\mathrm{NMR}-$ Spektrum ist identisch mit dem in der Literatur 5 beschriebenen.

Wir danken der Deutschen Forschungsgemeinschaft und dem Fonds der Chemischen Industrie für die finanzielle Unterstützung dieser Arbeit.

10 Spaltrohrkolonne HMS 300 der Fa. Fischer, BonnBad Godesberg.

11 M. Baudler, B. Carlsohn, W. Böhm u. G. ReuSCHENBACH, Z. Naturforsch. 31 b, 558 [1976].

12 L. R. Sмith u. J. L. Mills, Chem. Commun. 1974, 808.

13 M. Baudler, Ch. Pinner, Ch. Gruner, J. HellManN u. M. Schwamborn, Veröffentlichung in Vorbereitung.

14 G. A. Olah u. C. W. McFarland, J. Org. Chem. 34, 1832 [1969]; H. SchumanN, O. Stelzer u. U. Niederreuther, J. Organomet. Chem. 16, P 64 [1969]; Ch. A. Tolman, J. Amer. Chem. Soc. 92, 2956 [1970].

15 M. Baudler u. Ch. Gruner, Z. Naturforsch. 31 b, 1311 [1976].

162 reagiert mit $t-\mathrm{BuPCl}_{2}$ in siedendem Dioxan (35 Min.) bzw. siedendem Monoglyme (10 Min.) nicht nachweisbar zu 1 (M. BAUdLER u. CH. GRUNER, unveröffentlicht). 